1

\title{
sPepFinder expedites genome-wide identification of small proteins in bacteria
}

\section{6} 7

8

9

10

1. Department of Biological Chemistry, School of Medicine, University of California Irvine, Irvine, CA 92697, United States

2. Department of Molecular Biology and Microbiology, Tufts University School of Medicine.

$$
\text { Boston, MA 02111, United States }
$$

\author{
Lei $\mathrm{Li}^{1}$, Yanjie Chao ${ }^{2, *}$
}




\section{ABSTRACT}

25 Small proteins shorter than 50 amino acids have been long overlooked. A number of small

26 proteins have been identified in several model bacteria using experimental approaches and 27 assigned important functions in diverse cellular processes. The recent development of 28 ribosome profiling technologies has allowed a genome-wide identification of small proteins 29 and small ORFs (smORFs), but our incomplete understanding of small proteins hinders de 30 novo computational prediction of sm0RFs in non-model bacterial species. Here, we have 31 identified several sequence features for sm0RFs by a systematic analysis of all the known 32 small proteins in E. coli, among which the translation initiation rate is the strongest 33 determinant By integrating these features into a support vector machine learning model, 34 we have developed a novel sPepFinder algorithm that can predict conserved smORFs in 35 bacterial genomes with a high accuracy of $92.8 \%$. De novo prediction in E. coli has revealed 36 several novel sm0RFs with evidence of translation supported by ribosome profiling. 37 Further application of sPepFinder in 549 bacterial species has led to the identification of $38>100,000$ novel sm0RFs, many of which are conserved at the amino acid and nucleotide 39 levels under purifying selection. Overall, we have established sPepFinder as a valuable tool 40 to identify novel smORFs in both model and non-model bacterial organisms, and provided a 41 large resource of small proteins for functional characterizations. 


\section{INTRODUCTION}

51 Small proteins less than 50 amino acids have been increasingly appreciated to play critical

52 roles in diverse biological processes in all three kingdoms of life (Andrews and Rothnagel,

53 2014). Despite lacking annotations in bacterial genomes, small proteins encoded by open

54 reading frames (smORFs) have been fortuitously identified in the gaps between protein-

55 coding genes, sometime even inside small noncoding RNAs (Jørgensen et al., 2013; Pinel-

56 Marie et al., 2014). Most of the known bacterial small proteins are leader peptides upstream

57 of a structural gene, or interaction partners of larger protein (complexes), or small toxic

58 proteins as part of the toxin/antitoxin system (TA system) (Hemm et al., 2008). The

59 functions of those bacterial small proteins have been assigned in a myriad of physiological

60 pathways including biofilm formation, stress response, antibiotic resistance, virulence and

61 beyond (Duval and Cossart, 2017; Storz et al., 2014).

The identification of SmORFs has been challenging due to their small size and a lack of sequence/structural characteristics. Traditionally, bacterial small proteins were identified individually by biochemical isolation (Levin et al., 1993). With increasing availability of complete genome sequences, smORFs have been considered as 'noise' and intentionally left out during genomic annotation of ORFs by automatic computational programs. Very few tools were able to accurately predict sm0RFs directly from genome sequence (Baek et al., 2017; Boekhorst et al., 2011; Pauli et al., 2015; Zhu and Gribskov, 2019). It was only recently that a combination of computational screening and experimental approaches identified an array of small proteins in the model bacterium $E$. coli (Hemm et al., 2008) (Miravet-Verde et al., 2019).

The recently developed ribosomal profiling approach (a.k.a. Ribo-seq of ribosomeprotected mRNA fragments) has become instrumental to detect novel ORFs in both eukaryotic and prokaryotic organisms (Brar and Weissman, 2015; Ingolia, 2014; Weaver et al., 2019). Ribo-seq data could provide evidence of active translation, enabling straightforward predictions of novel ORFs as well as smORFs with high confidence (Aspden et al., 2014; Bazzini et al., 2014; Calviello et al., 2016; Ji et al., 2015; Raj et al., 2016). However, 
81 species such as E. coli and Bacillus subtilis (Li et al., 2012; Oh et al., 2011; Sberro et al., 2019;

82 Schrader et al., 2014; Woolstenhulme et al., 2015). The low resolution of Ribo-seq analysis

83 in bacteria complicates the precise determination of reading frames and starting codons.

84 The resolution could be improved using some modified protocols (Weaver et al., 2019),

85 which however inevitably introduce additional biases and costs. Therefore, other

86 alternative approaches are in need to allow the rapid identification of smORFs in a wide

87 range of bacteria.

88 Here, we have identified three strong features of sm0RF that allow the discrimination of smORFs from random ORFs in bacterial chromosomes. We have developed upon these features a support vector machine learning model (sPepfinder) to predict smORF with $92.8 \%$ accuracy. sPepfinder can be used as a stand-alone tool for the prediction of smORFs from genome sequences with high-confidence, or used in combination with ribosome profiling to accurately predict translating sm0RFs. The development of sPepfinder promises a high-throughput identification of Sm0RFs in diverse bacterial species for future functional studies.

\section{RESULTS}

\section{Systematic analyses revealed distinct features for smORF}

99 Most small proteins lack functional domains and remain uncharacterized. To better 100 understand the features of small proteins, we sought to systematically analyse 94 known 101 small proteins in Escherichia coli from EcoGene database (version: 3.0) (Zhou and Rudd, 102 2012). These small proteins can be classified into several categories based on genetic 103 locations. Most of the small proteins are encoded by independent genes in the intergenic 104 regions. Less than a quarter partially overlap with other genes or pseudogenes, and only $1057 \%$ are leader peptides linked with the gene downstream. A vast majority of known small 106 proteins (96\%) use a canonical start codon ATG or GTG, with an average length of 30 amino 107 acids (Figure 1A). Interestingly, our analysis of amino acid frequency revealed that three 108 amino acids are significantly enriched in small proteins (student $t$ test: $p$-value $<0.001$, 109 Figure 1B). All three amino acids (Leucine, Isoleucine and Valine) are hydrophobic amino 110 acids, in line with previous observations that many small proteins are localized in the 111 membrane (Alix and Blanc-Potard, 2009; Storz et al., 2014). 
The ribosome binding site (RBS) is the key sequence element that determines the

114 translation efficiency of proteins. To understand the translation efficiency of small proteins

115 in E. coli, we have analysed their RBS strength and translation initiation rate using a 116 thermodynamic model, which evaluates the Gibbs free energy of molecular interactions

117 between RBS and the 16S ribosomal RNA (Salis et al., 2009). Strikingly, we found that most

118 small proteins possess stronger RBS with significantly higher translation initiation scores,

119 compared to that of random ORFs predicted in the intergenic regions (student $t$ test: $p$ 120 value: 0.001885 , Figure $1 \mathrm{C}$ ). In fact, the top quartile of known small proteins has stronger

121 RBS than most, if not all, the intergenic short ORFs. Further analysis showed that weaker

122 RBS (bottom quartile) are often associated with small toxin proteins and anti-toxins (TA

123 systems), including ldrA, ldrB, ldrC, whose functions may require lower expression (Fozo et

124 al, 2008).

125

sPepfinder employs a machine learning model to predict smORFs

127 Having identified these features for small proteins, we next established a machine learning 128 algorithm to model on these features and predict new sm0RFs (Figure 2A). Specifically, a 129 support vector model was built by training on a positive dataset that contains 110 non130 redundant known small proteins collected from ten different Gamma-proteobacterial 131 species including E. coli, and a negative dataset that contains 136 short ORF predicted in 132 rRNAs and tRNAs in these representative strains (Supplemental Table S1). The input 133 parameters included the RBS strength (the translation initiation score), the frequency of 134 hydrophobic amino acids (I, L, V), and their coding potential scores based on the sequence 135 conservation of codons (RNAcode, (Washietl et al., 2011).

In order to evaluate the performance of our algorithm, we have carried out a ROC 138 (Receiver operating characteristic) analysis with 10-fold cross-validation (Figure 2B). The 139 training sets were split into ten datasets, and then $90 \%$ of them was used a training model 140 and the remaining $10 \%$ as test datasets. The ROC results show that our SVM model reached $14192.8 \%$ accuracy on predicting the true-positives and the true-negatives. Among all three 142 features of small proteins, the translation initiation score is the most predominant SVM 
143 classifier (Figure 2B). Thus, the RBS strength is the strongest indicator of prediction

144 confidence, whereas the additional sequence features help define the frame of translation

145 and the size of small proteins.

\section{De novo prediction of SmORFs in Escherichia coli}

148 To further validate our approach, we have used sPepfinder to predict sm0RFs in the

149 genome of Escherichia coli MG1655, and evaluated the expression of small proteins using 150 the available ribosome profiling data in the GWIPS-viz database (Michel et al., 2014). In 151 total, sPepfinder has found 124 smORFs using a SVM score cutoff at probability of 0.9

152 (Supplemental Table S2). Interestingly, more than 40\% of them are known small proteins 153 (Figure 3A), including several known small proteins that were not included in our initial 154 training set such as Yoh0, a known membrane-bound small protein (Hemm et al., 2008). 155 sPepfinder has successfully predicted the correct size and the reading frame of Yoh0 156 (Figure 3B), highlighting the accuracy of our SVM model. also novel smORFs in the 5'UTRs and 3'UTRs (Figure 3A), many of which are supported by ample amount of ribosome footprints as well as RNA-seq reads at different conditions.

161 These include an sm0RF downstream of $y f j T$ (Figure 3C), a putative u0RF (Fig. 3D) in the 162 5'UTR of $d b p A$ encoding an ATP-dependent RNA helicase (Diges \& Uhlenbeck, 2005), as well 163 as an smORF in the 3'UTR of yciX (Fig. 3E). The latter 31aa-smORF is conserved across 164 enterobacteria (Figure 3G), and is encoded on the highly expressed yciX 3'UTR without an 165 independent promoter (Figure 3F), according to the available differential RNA-seq data 166 (Thomason et al., 2015).

\section{Genome-wide prediction of SmORFs in diverse bacteria}

169 The successful application of sPepfinder in E. coli has promoted us to predict smORF in 170 other related bacterial species. Using the same strict cutoff (probability $>0.9$ ), we have 171 harnessed sPepfinder to identify smORFs in 549 bacterial genomes representing the full

172 spectrum of Proteobacteria phylum (Supplemental Table 3), including numerous human 173 pathogens such as Salmonella, Vibrio and Pseudomonas spp. This systematic analysis has 
174 identified a total of 108, 871 sm0RFs (Supplemental Table 4), presenting an atlas of small

175 proteins in bacteria and a tremendous increase compared to the number of known sm0RFs

176 to date. We have predicted a large number of smORFs in all five classes of Proteobacteria

177 (Figure 4A), whereas the highest number of sm0RFs were found among Gamma-

178 Proteobacteria ( 200 per genome).

To better understand these novel smORFs, we have analyzed the conservation 181 profiles for all 1235 smORFs identified in the Enterobacteriaceae family (Supplemental 182 Table 5), blasted against a panel of 57 representative Enterobacterial species. As depicted 183 in Figure 4B, these sm0RFs are separated into three major groups. The first group with 184 highest conservation includes ribosomal associated small proteins such as RpmJ, as well as 185 a number of known proteins such as AcrZ, YobF, LeuL, CydX and SecY. For example, CydX 186 has been recently characterized as a conserved component of the cytochrome bd oxidase complex (Allen et al., 2014; Ramamurthi and Storz, 2014). In this group, we have identified several novel small proteins (Supplemental Table 5), whose high conservation indicates their functional importance and warrants future studies in the native bacterial strains. The second group shows somewhat intermediate conservation profiles, including small proteins identified in a range of closely-related bacterial species (Figure 4B). The third group only displays conservation patterns within one or two species, such as the strains of Klebsiella or Erwinia. A few putative uORFs were included in this group, indicating species-specific functions linked to some unique genes.

We further investigated the conservation patterns by analysing the selection pressure on codon usage (Figure 4C). For this, we have performed a Ka/Ks ratio analysis on the predicted smORFs in Escherichia coli and Salmonella, which have an average length of 25 amino acids. The result revealed that most of the novel sm0RFs have a substantially lower $\mathrm{Ka} / \mathrm{Ks}$ ratio compared to the that of random ORFs in chromosome (Figure $4 \mathrm{C}$ ). These predicted sm0RFs have a $\mathrm{Ka} / \mathrm{Ks}$ ratio far below 1 , indicating a significant number of nucleotide sequence changes that do not result in changes in protein sequence. In other words, the novel smORFs are under purifying selection and likely to be functional, 204 bolstering the reliability of the sPepfinder algorithm in the prediction of novel smORFs. 


\section{DISCUSSION}

In this work, we presented a new computational approach sPepfinder that can rapidly and efficiently detect smORFs in a genome-wide manner. The sPepfinder utilizes the distinct features of bacterial small proteins, and achieves high accuracy and specificity. The application of sPepfinder in hundreds of bacterial strains has revealed the first atlas of small proteins in the Proteobacteria Phylum with over 0.1 million smORFs. With the burgeoning interest in the studies of smORFs in bacteria, the development of sPepfinder will significantly accelerate their experimental identification and characterization in a large variety of bacterial species.

sPepfinder will help identify potential smORFs in experimentally generated data, though it was initially developed for de novo prediction of smORFs from empty intergenic regions in genome sequences. The application of RNA-seq or ribosome profiling alone has shown promise to identify novel smORFs in model bacteria such as E. coli (Weaver et al., 2019). Starting from available experimental data, computational tools could more reliably predict ORFs in RNA fragments that are bound to translating ribosomes (Miravet-Verde et al., 2019; Ndah et al., 2017). A combination of sPepfinder with ribosome profiling and mass spectrometry will further enhance the accuracy of prediction, and allow the identification of functional small proteins that are highly conserved and expressed at specific physiological conditions, exemplified by our study in Salmonella typhimurium (Venturini et al., in 227 preparation).

The sPepfinder algorithm is not without limitations. To ensure the high accuracy of prediction, we have only considered the open reading frames starting with a canonical ATG or GTG codon. The prediction will inevitable lose a small number of proteins with other non-canonical start codons (Li et al., 2012), reducing the sensitivity of prediction while

233 maintaining a high specificity. Due to the reliance on the translation initiation score and the

234 binding strength of RBS, our model will not take into consideration the leaderless mRNAs, 235 though this group of mRNAs should be rare. In addition, our SVM model was trained on a 
236 collection of known small proteins in E. coli, therefore its performance in more distant-

237 related organisms will need to be further evaluated, when more experimental data become

238 available. The future inclusion of more experimentally verified small proteins will certainly

239 improve our SVM model for a better performance and wider application in diverse bacterial 240 species and complex bacterial communities, such as plant- and human-associated 241 microbiome.

MATERIAL AND METHODS

\section{Benchmark of small protein datasets}

247 We have downloaded the gene table with protein only from Ecogene 3.0 database (Zhou 248 and Rudd, 2012). In total 4323 genes were retrieved including 94 small proteins.

\section{The sPepfinder workflow}

251 The intergenic sequences and with 20 bp overlap with known annotation of each genome was used as a starting point. The $20 \mathrm{bp}$ upstream sequences of each sm0RF were extracted

253 and the translation initiation score was calculated using Ribosome binding site calculator 254 (Salis et al., 2009). The conservation analysis of these sm0RFs was performed to obtain the 255 sequence alignment, which was further used to define the coding potential scores by 256 RNAcode (Washietl et al., 2011). The frequency of every amino acid in small proteins and 257 control proteins was analysed, including the three hydrophobic amino acids (I, L, V) that 258 showed significant enrichment in known small proteins. Ribosome profiling sequencing 259 reads were analysed by including the center weighting steps to normalize ribosome pausing 260 sites. The in silico predicted sm0RFs are considered sm0RFs candidates when they show 261 evidence of translation based on ribosome footprinting data.

\section{Analysis of Ribo-seq data}

264 We overlapped our smORFs prediction result with the ribosome profiling data from 265 Escherichia coli using GWIPS-viz browser (Michel et al., 2014). 


\section{Nonsynonymous and synonymous substitutions (Ka/Ks ratio)}

268 The nucleotides and peptide sequences of small peptides in Escherichia coli and Salmonella

269 Typhimurium were analysed using the KaKs calculator software (Zhang et al., 2006) to 270 examine nonsynonymous (Ka) and synonymous (Ks) substitutions and the resulting and $271 \mathrm{Ka} / \mathrm{Ks}$ values, using the approximate method ' $\mathrm{NG}$ '.

\section{ACKNOWLEDGEMENT}

276 We thank Konrad Förstner for his initial input and suggestions, and Jörg Vogel for critical reading and comments on the manuscript.

AUTHOR CONTRIBUTIONS

L.L., Y.C. conceived and supervised this project. L.L. performed the data analyses. L.L., Y.C. interpreted the data and wrote the manuscript.

\section{CONFLICT OF INTEREST}

284 The authors declare no competing financial interests.

\section{REFERENCES}

289 Alix, E., and Blanc-Potard, A. (2009). Hydrophobic peptides: novel regulators within bacterial membrane. Mol Microbiol 72, 5-11.

Andrews, S., and Rothnagel, J. (2014). Emerging evidence for functional peptides encoded by short open reading frames. Nat Rev Genet 15, 193-204.

293 Aspden, J., Eyre-Walker, Y., Phillips, R., Amin, U., Mumtaz, M., Brocard, M., and Couso, J. 294 (2014). Extensive translation of small Open Reading Frames revealed by Poly-Ribo-Seq. 295 Elife 3, e03528.

296 Baek, J., Lee, J., Yoon, K., and Lee, H. (2017). Identification of Unannotated Small Genes in 
298 Bazzini, A., Johnstone, T., Christiano, R., Mackowiak, S., Obermayer, B., Fleming, E., Vejnar, C., 299 Lee, M., Rajewsky, N., Walther, T., et al. (2014). Identification of small ORFs in vertebrates 300 using ribosome footprinting and evolutionary conservation. Embo J 33, 981-993.

301 Boekhorst, J., Wilson, G., and Siezen, R. (2011). Searching in microbial genomes for encoded 302 small proteins. Microb Biotechnol 4, 308-313.

303 Brar, G., and Weissman, J. (2015). Ribosome profiling reveals the what, when, where and 304 how of protein synthesis. Nat Rev Mol Cell Bio 16, 651-664.

305 Calviello, L., Mukherjee, N., Wyler, E., Zauber, H., Hirsekorn, A., Selbach, M., Landthaler, M., 306 Obermayer, B., and Ohler, U. (2016). Detecting actively translated open reading frames in 307 ribosome profiling data. Nat Methods 13, 165-170.

308 Duval, M., and Cossart, P. (2017). Small bacterial and phagic proteins: an updated view on a 309 rapidly moving field. Curr Opin Microbiol 39, 81-88.

310 Hemm, M., Paul, B., Schneider, T., Storz, G., and Rudd, K. (2008). Small membrane proteins 311 found by comparative genomics and ribosome binding site models. Mol Microbiol 70, 14873121501.

313 Ingolia, N. (2014). Ribosome profiling: new views of translation, from single codons to 314 genome scale. Nat Rev Genet 15, 205-213.

315 Ji, Z., Song, R., Regev, A., and Struhl, K. (2015). Many lncRNAs, 5’UTRs, and pseudogenes are 316 translated and some are likely to express functional proteins. Elife 4, e08890.

317 Jørgensen, M., Thomason, M., Havelund, J., Valentin-Hansen, P., and Storz, G. (2013). Dual 318 function of the McaS small RNA in controlling biofilm formation. Gene Dev 27, 1132-1145.

319 Levin, P., Fan, N., Ricca, E., Driks, A., Losick, R., and Cutting, S. (1993). An unusually small 320 gene required for sporulation by Bacillus subtilis. Mol Microbiol 9, 761-771.

321 Li, G., Oh, E., and Weissman, J. (2012). The anti-Shine-Dalgarno sequence drives 
322 translational pausing and codon choice in bacteria. Nature 484, 538-541.

323 Michel, A., Fox, G., Kiran, A., Bo, C., O’Connor, P., Heaphy, S., Mullan, J., Donohue, C., Higgins, 324 D., and Baranov, P. (2014). GWIPS-viz: development of a ribo-seq genome browser. Nucleic 325 Acids Res 42, D859-D864.

326 Miravet-Verde, S., Ferrar, T., Espadas-García, G., Mazzolini, R., Gharrab, A., Sabido, E., 327 Serrano, L., and Lluch-Senar, M. (2019). Unraveling the hidden universe of small proteins in 328 bacterial genomes. Mol Syst Biol 15, e8290.

329 Ndah, E., Jonckheere, V., Giess, A., Valen, E., Menschaert, G., and Van Damme, P. (2017). 330 REPARATION: ribosome profiling assisted (re-)annotation of bacterial genomes. Nucleic 331 Acids Res 45, gkx758-.

332 Oh, E., Becker, A., Sandikci, A., Huber, D., Chaba, R., Gloge, F., Nichols, R., Typas, A., Gross, C., 333 Kramer, G., et al. (2011). Selective Ribosome Profiling Reveals the Cotranslational 334 Chaperone Action of Trigger Factor In Vivo. Cell 147, 1295-1308.

335 Pauli, A., Valen, E., and Schier, A. (2015). Identifying (non-)coding RNAs and small peptides: 336 Challenges and opportunities. Bioessays 37, 103-112.

337 Pinel-Marie, M., Brielle, R., and Felden, B. (2014). Dual Toxic-Peptide-Coding Staphylococcus 338 aureus RNA under Antisense Regulation Targets Host Cells and Bacterial Rivals Unequally. 339 Cell Reports 7, 424-435.

340 Raj, A., Wang, S., Shim, H., Harpak, A., Li, Y., Engelmann, B., Stephens, M., Gilad, Y., and 341 Pritchard, J. (2016). Thousands of novel translated open reading frames in humans inferred 342 by ribosome footprint profiling. Elife 5, e13328.

343 Salis, H., Mirsky, E., and Voigt, C. (2009). Automated design of synthetic ribosome binding 344 sites to control protein expression. Nat Biotechnol 27, 946-950.

345 Sberro, H., Fremin, B., Zlitni, S., Edfors, F., Greenfield, N., Snyder, M., Pavlopoulos, G., 346 Kyrpides, N., and Bhatt, A. (2019). Large-Scale Analyses of Human Microbiomes Reveal 347 Thousands of Small, Novel Genes. Cell 178, 1245-1259.e14. 
348 Schrader, J., Zhou, B., Li, G., Lasker, K., Childers, W., Williams, B., Long, T., Crosson, S., 349 McAdams, H., Weissman, J., et al. (2014). The Coding and Noncoding Architecture of the 350 Caulobacter crescentus Genome. Plos Genet 10, e1004463.

351 Storz, G., Wolf, Y., and Ramamurthi, K. (2014). Small Proteins Can No Longer Be Ignored. 352 Annu Rev Biochem 83, 753-777.

353 Thomason, M., Bischler, T., Eisenbart, S., Förstner, K., Zhang, A., Herbig, A., Nieselt, K., 354 Sharma, C., and Storz, G. (2015). Global Transcriptional Start Site Mapping Using Differential 355 RNA Sequencing Reveals Novel Antisense RNAs in Escherichia coli. J Bacteriol 197, 18-28.

356 Washietl, S., Findeiß, S., Müller, S., Kalkhof, S., Bergen, M., Hofacker, I., Stadler, P., and 357 Goldman, N. (2011). RNAcode: Robust discrimination of coding and noncoding regions in 358 comparative sequence data. Rna 17, 578-594.

359 Weaver, J., Mohammad, F., Buskirk, A., and Storz, G. (2019). Identifying Small Proteins by 360 Ribosome Profiling with Stalled Initiation Complexes. Mbio 10, e02819-18.

361 Woolstenhulme, C., Guydosh, N., Green, R., and Buskirk, A. (2015). High-Precision Analysis 362 of Translational Pausing by Ribosome Profiling in Bacteria Lacking EFP. Cell Reports 11, 363 13-21.

364 Zhang, Z., Li, J., Zhao, X., Wang, J., Wong, G., and Yu, J. (2006). KaKs_Calculator: Calculating Ka 365 and Ks Through Model Selection and Model Averaging. Genom Proteom Bioinform 4, 259366263.

367 Zhou, J., and Rudd, K. (2012). EcoGene 3.0. Nucleic Acids Res 41, D613-D624.

368 Zhu, M., and Gribskov, M. (2019). MiPepid: MicroPeptide identification tool using machine 369 learning. Bmc Bioinformatics 20, 559. 
bioRxiv preprint doi: https://doi.org/10.1101/2020.05.05.079178; this version posted May 5, 2020. The copyright holder for this preprint (which was not certified by peer review) is the author/funder, who has granted bioRxiv a license to display the preprint in perpetuity. It is made available under aCC-BY-NC-ND 4.0 International license.

373 
376 Figure 1. Bacterial sm0RFs have distinct sequence features. (A) Size distribution of known small proteins in Escherichia coli. (B) The percentage of hydrophobic amino acids in small

378 proteins. The negative control (NE) are random short ORFs in the chromosome. (C) The translation initiation score for known small proteins and random intergenic short ORFs in $E$. coli.

Figure 2. The identification of bacterial sm0RFs using sPepfinder. (A) Schematic framework of the sPepfinder algorithm. (B) ROC evaluation of sPepfinder performance with different features, including TIS (transcription initiation score), coding conservation potential and hydrophobic amino acid frequency.

Figure 3. sPepfinder identifies 104 sm0RFs in Escherichia coli. (A) Pie chart distribution of 124 predicted smORFs in Escherichia coli. (B) The Ribo-seq and mRNA-seq coverage profiles for the known small protein Yoh0, shown as a red bar. Black bars indicate alternative frames of translation. (C-E) The Ribo-seq and mRNA-seq coverage profiles for new small proteins sm0RF88 (C), sm0RF37 (D), and sm0RF34 (E). (F) differential RNA-seq profiles for the transcripts of sm0RF34. TEX+/- indicates the treatment of 5'p-dependent terminator exoribonuclease. (G)The sequence conservation of sm0RF34 among the closerelated enterobacterial species.

Figure 4. The landscape of sm0RFs candidates in the Proteobacteria phylum. (A) Barplot showing the average number of sm0RFs predicted per genome among Proteobacteria. (B) Heatmap showing the conservation profiles of sm0RFs in 57 representative Enterobacteria strains. Values in the heatmap represent the percentage of conservation of each sm0RF. (C) The distribution of $\mathrm{Ka} / \mathrm{Ks}$ ratios for the known small proteins, predicted novel smORFs, and random ORF as background in Escherichia coli and Salmonella. 


\section{SUPPLEMENTAL TABLES}

406

407 Table S1. The training datasets used for sPepfinder. The SVM model was trained using a 408 positive dataset of 123 known small proteins, and a negative dataset including 136 short 409 ORFs predicted in rRNA and tRNA regions.

410

411 Table $\mathbf{S 2}$. The list of 124 sm0RFs predicted in E. coli by sPepfinder.

412

413 Table S3. The list of all the 549 proteobacterial genomes used for prediction.

415 Table S4. The complete list of all the predicted smORFs in Proteobacteria.

417 Table S5. The list of the conserved sm0RFs in 57 Enterobacteria. 
bioRxiv preprint doi: https://doi.org/10.1101/2020.05.05.079178; this version posted May 5, 2020. The copyright holder for this preprint (which was not certified by peer review) is the author/funder, who has granted bioRxiv a license to display the preprint in perpetuity. It is made available under aCC-BY-NC-ND 4.0 International license.

\section{$421 \quad$ Figure 1}

422

A

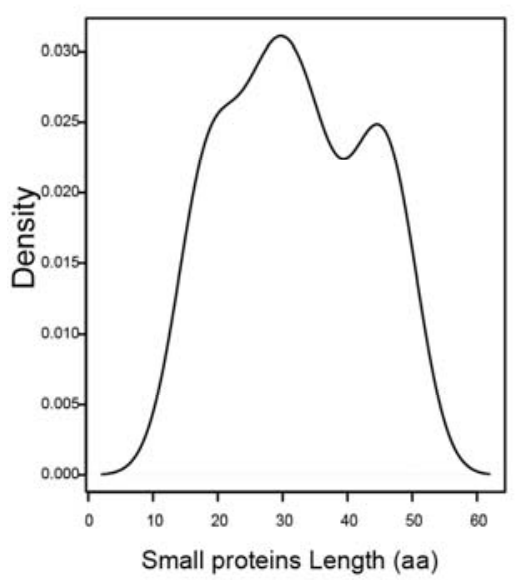

B

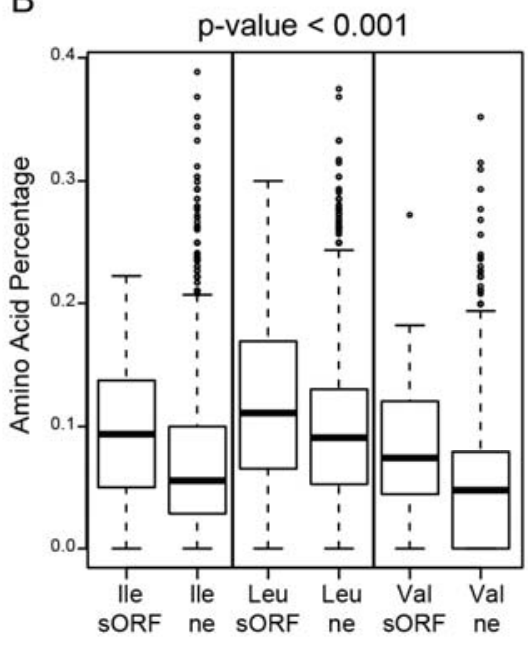

C

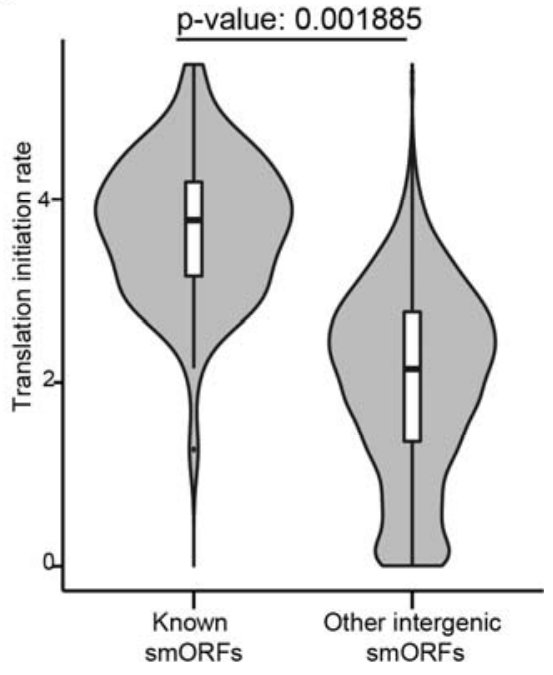


bioRxiv preprint doi: https://doi.org/10.1101/2020.05.05.079178; this version posted May 5, 2020. The copyright holder for this preprint (which

was not certified by peer review) is the author/funder, who has granted bioRxiv a license to display the preprint in perpetuity. It is made available under aCC-BY-NC-ND 4.0 International license.

\section{$425 \quad$ Figure 2}

426

A

427

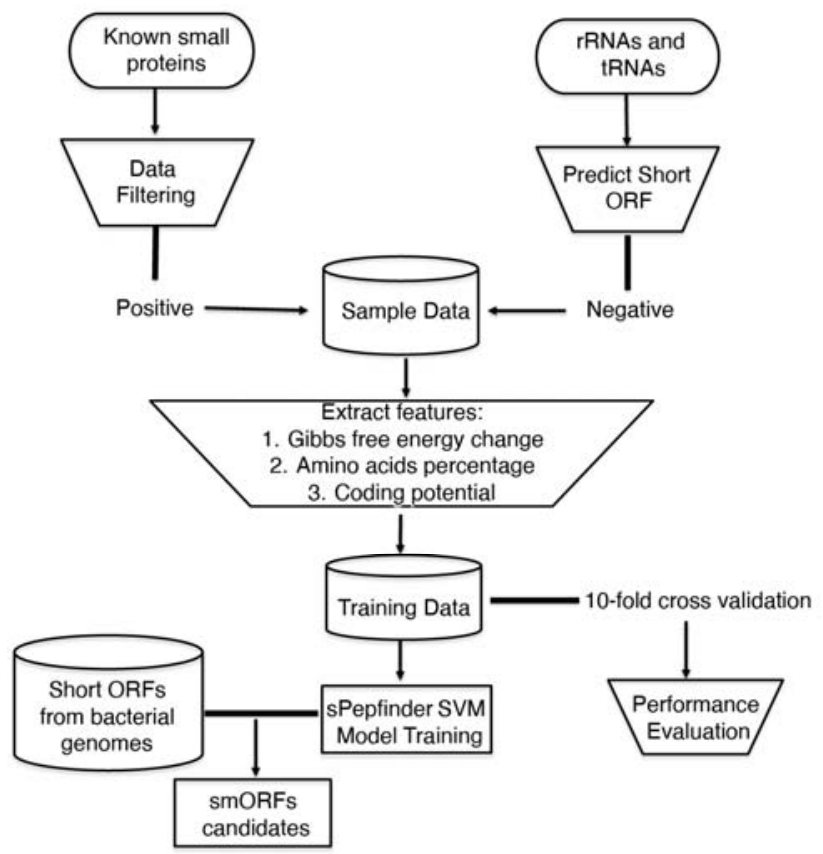

B

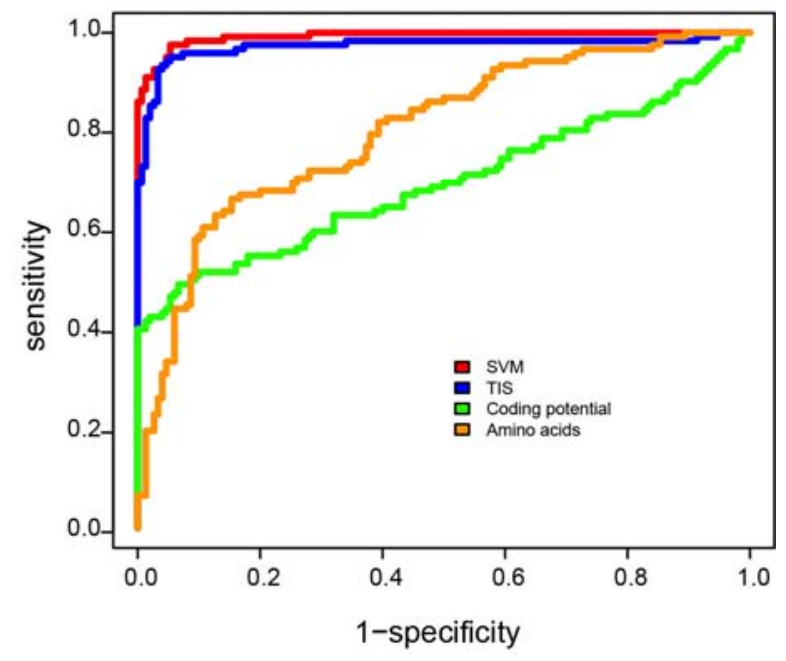

428 
bioRxiv preprint doi: https://doi.org/10.1101/2020.05.05.079178; this version posted May 5, 2020. The copyright holder for this preprint (which was not certified by peer review) is the author/funder, who has granted bioRxiv a license to display the preprint in perpetuity. It is made available under aCC-BY-NC-ND 4.0 International license.

\section{$429 \quad$ Figure 3}

A

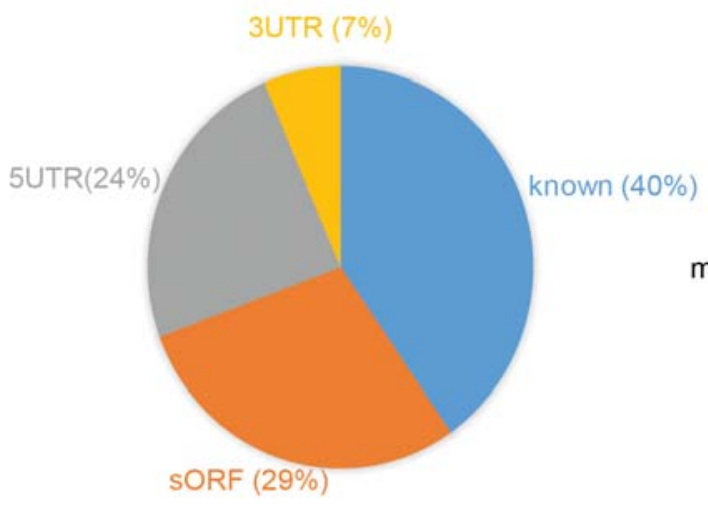

Escherichia coli (124)

C

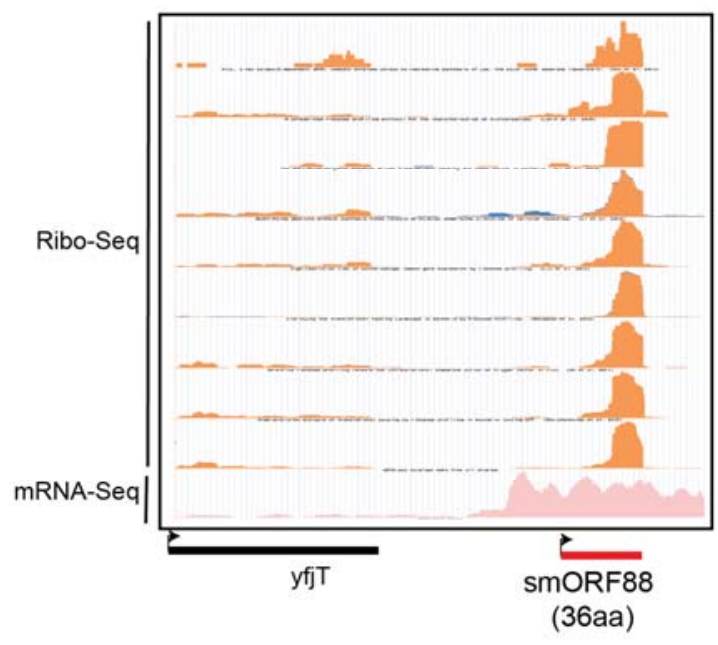

E

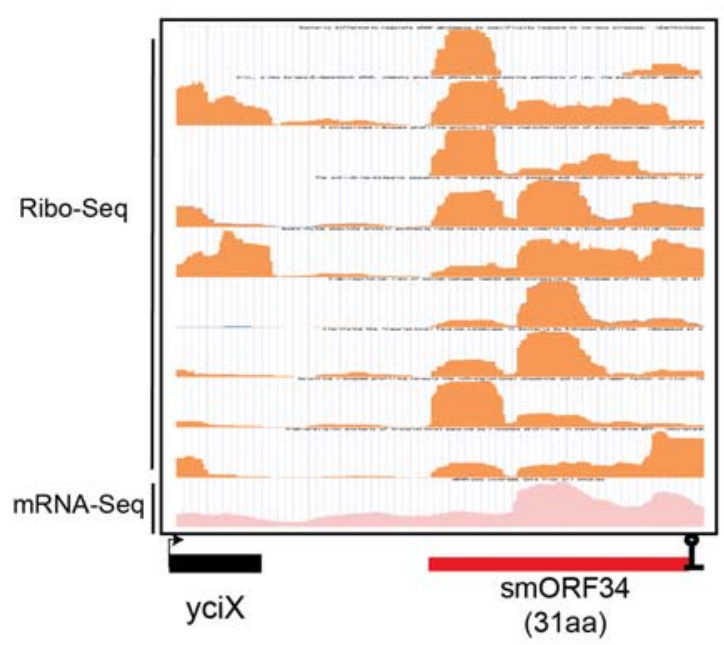

B

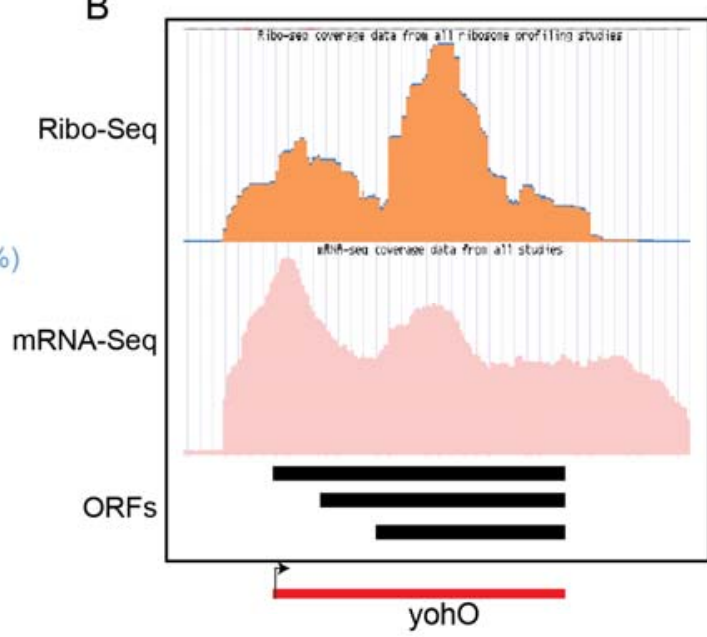

D

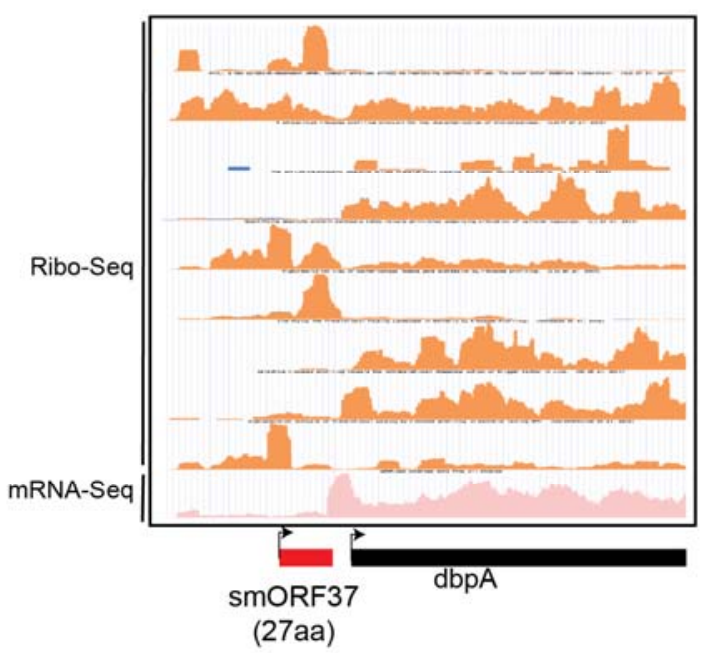

$\mathrm{F}$

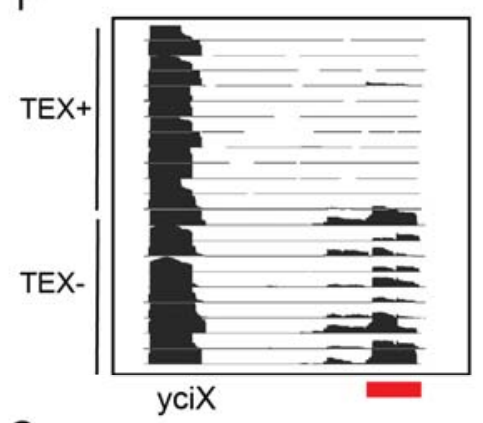

G

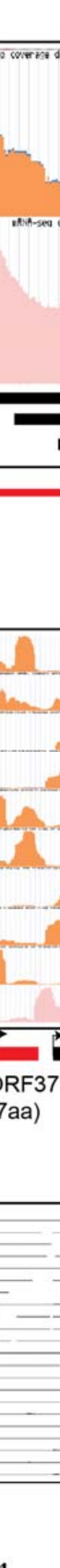

$1 \quad 10 \quad 20$

3033

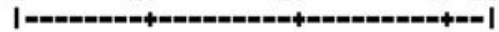

Escherichia MINT--NMKYMSHMGAFSLSHLFMAELLUIITH

Shigella MINT--NMKYMSHMGRFSLSMLFMAELLUIITH

Salnonella MLTS--NIKYUSHTGRFILSLLFHAELIUI

Enterobacter MMNTLQNYKYHSHMGPFTYSTLFHCQLIML

Consensus M.nt..N.KYHSHnGifF.1S.LFHa"\#LiHi... 
bioRxiv preprint doi: https://doi.org/10.1101/2020.05.05.079178; this version posted May 5, 2020. The copyright holder for this preprint (which was not certified by peer review) is the author/funder, who has granted bioRxiv a license to display the preprint in perpetuity. It is made available under aCC-BY-NC-ND 4.0 International license.

\section{$431 \quad$ Figure 4}

432

A

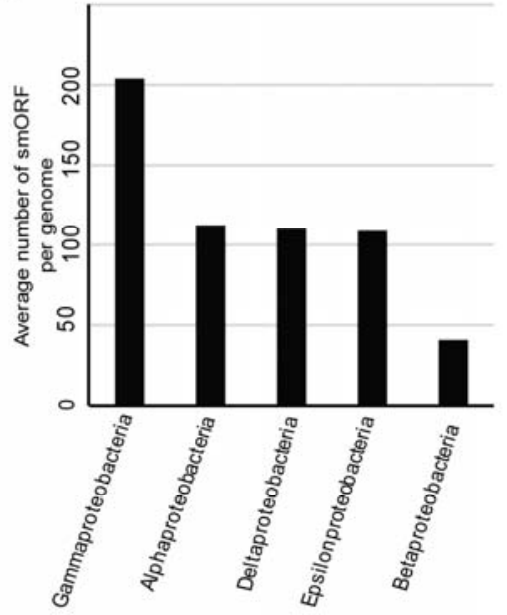

C

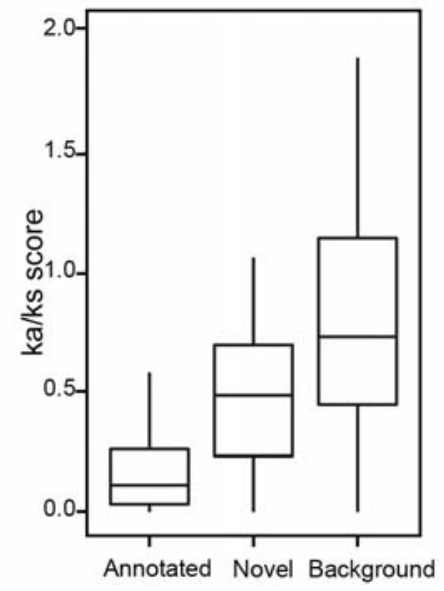

B

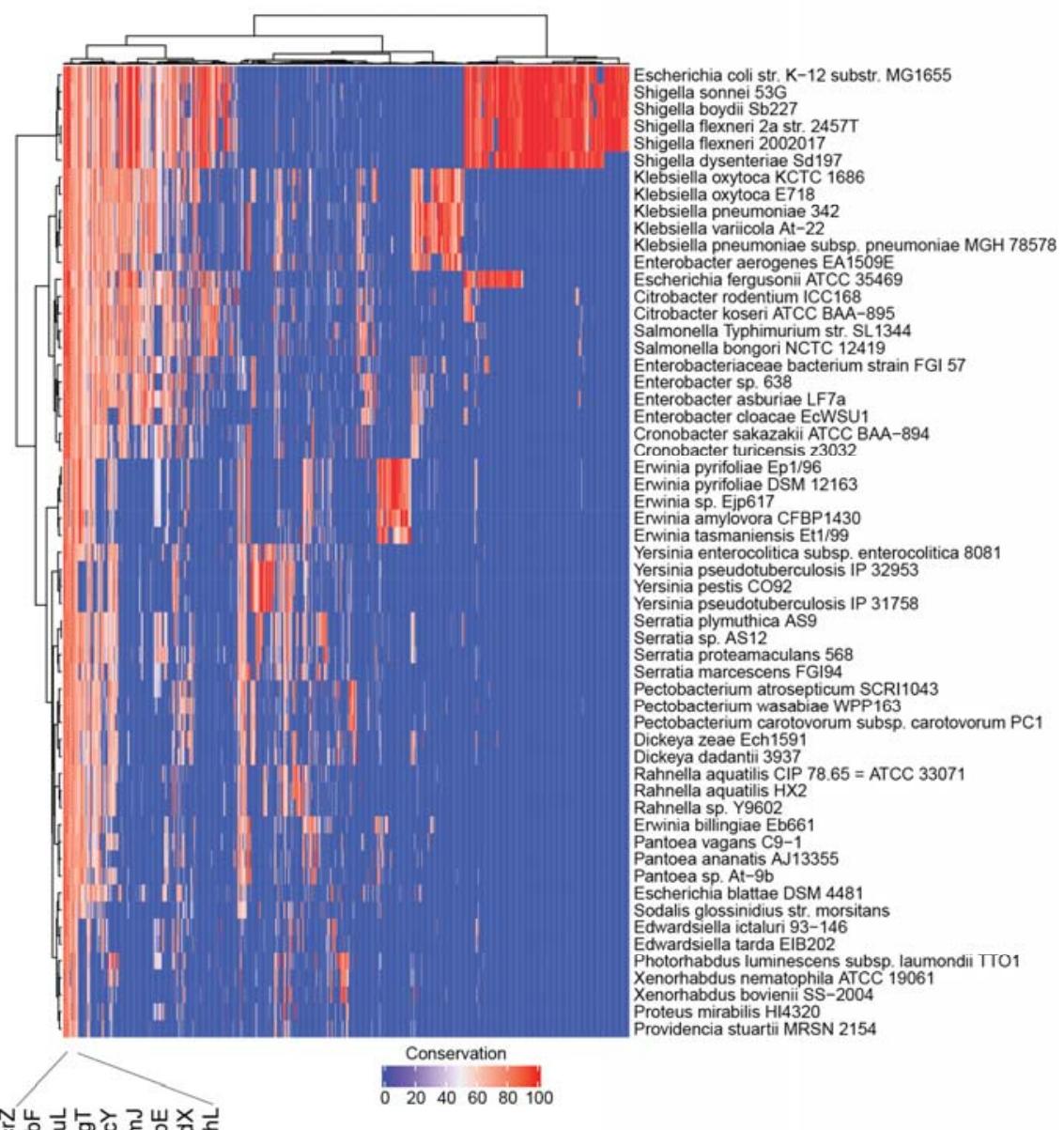

